\title{
Self-association of double-hydrophilic copolymers of acrylic acid and poly(ethylene oxide) macromonomer
}

\author{
Eric Khousakoun, Jean-François Gohy Robert Jérôme \\ Center for Education and Research on Macromolecules (CERM), University of Liège, Sart-Tilman, B6, 4000 \\ Liège, Belgium
}

\begin{abstract}
Poly(ethylene oxide) (PEO) end-capped by a methacrylate unsaturation was copolymerized with acrylic acid by RAFT with dibenzyltrithiocarbonate as a chain transfer agent. Tapered triblock copolymers consisting of a poly(acrylic acid) (PAA) inner block and comb-like outer blocks of PEO macromomers were formed as result of the comonomers reactivity ratios. Composition of these copolymers and length of the PEO branches were varied. Dynamic light scattering (DLS) was used to characterize the aggregates formed in water and to investigate their response to stimuli, such as $\mathrm{pH}$, temperature and ionic strength. In parallel, the morphology of the aggregates was directly observed by transmission electron microscopy (TEM). Well-defined aggregates were formed in the $5<\mathrm{pH}<8$ range, with a morphology strongly depending on the copolymer composition. At $\mathrm{pH}<5$, the copolymers were poorly soluble and no well-defined structure was observed, whereas free chains were formed at $\mathrm{pH}>8$ as consequence of the complete ionization of the PAA block.
\end{abstract}

Keywords: Self-association; Double-hydrophilic block copolymers; Stimuli-responsive copolymers

\section{Introduction}

Increasing attention is paid nowadays to double hydro-philic copolymers, as recently reviewed by Cölfen [1]. These copolymers consist of two blocks soluble in water, although able to form aggregates or micelles in aqueous solution under certain conditions. For instance, one of the two blocks can be made hydrophobic by an appropriate change of $\mathrm{pH}$ or temperature, with formation of an amphiphilic copolymer prone to self-association in water. This behavior is typical of poly(2-vinylpyridine)-block-poly(ethylene oxide) copolymers that exist as unimers at $\mathrm{pH}<5$ and form micelles at higher $\mathrm{pH}$ [2]. In an alternative approach, the water-soluble blocks are selected for mutual non-covalent interactions, such as electrostatic interactions and hydrogen-bonding, which can lead to the formation of well-defined aggregates. Aggregation driven by hydrogen-bonding is typically illustrated by double hydrophilic block copolymers of poly(ethylene oxide), PEO, and poly-(meth)acrylic acid, $\mathrm{P}(\mathrm{M}) \mathrm{AA}$. Complexation of EO and (M)AA units was originally used to tune the rheological properties of aqueous solutions $[3,4]$. However, the sensitivity of these interactions to $\mathrm{pH}$ and temperature, makes EO and (M)AA containing copolymers stimuli-responsive. Statistical copolymers of MAA and PEO macromonomers have been reported as smart $\mathrm{pH}$-respon-sive stabilizers for emulsions [5]. $\mathrm{pH}$-responsive crosslinked nanospheres of PMAA-g-PEO copolymers have also been designed for controlled drug release purpose [6], whereas PMAA$b$-PEO copolymers with a longer PEO block form well-defined $\mathrm{pH}$ - and temperature-responsive micelles [7]. It must be noted that in the aforementioned examples, MAA was used rather than AA as the H-donor moiety. However, the methyl substituent of MAA can favor hydrophobic interactions and the aqueous solutions show a more complex behavior as assessed by a cloud-point [8]. Moreover, the possible role of the architecture of the copolymers on self-association has not been investigated extensively. In this respect, the work by Holappa et al. [9] must be mentioned for the comparison of linear and graft copolymers of PEO and (M)AA. However, the structure of graft copolymers was ill-defined, and the morphology of the aggregates formed in water was not investigated.

This paper aims at reporting on the copolymerization of mixtures of AA and poly(ethylene oxide)-methyl-ethermethacrylate (PEOMA) macromonomers by reversible addition fragmentation chain transfer (RAFT) with a difunctional chain transfer agent. According to the reactivity ratios determined elsewhere (Table 1) and the control on the chain growth (as is the case for RAFT of (meth)acrylic compounds), tapered triblocks must be formed with a inner PAA block and two outer comb-like blocks of PEOMA (Scheme 1). Composition and length of the PEOMA branches have been changed, and the aggregates formed in water have been analyzed by dynamic light scattering (DLS) and observed by transmission electron microscopy (TEM) in relation to $\mathrm{pH}$, temperature and ionic strength. 
Table 1 Molecular characteristic features of the synthesized (co)polymers

\begin{tabular}{|c|c|c|c|c|c|}
\hline (co)polymers & Acronym & $\mathbf{D P}_{\mathrm{AA}}$ & DPP $_{\text {EOMA }}$ & ${ }^{\Gamma}$ AA $/{ }^{T}$ PEOMA $^{\text {a }}$ & $M_{n}\left({ }^{1}\right.$ H NMR $)$ \\
\hline $\mathrm{P}\left(\mathrm{PEO}_{11} \mathrm{MA}\right)_{12}-b-$ & 1 & 356 & 12 & $0.36 / 2.81$ & 32,000 \\
\hline \multicolumn{6}{|l|}{$\mathrm{PAA}_{356}$} \\
\hline $\begin{array}{l}\mathrm{P}\left(\mathrm{PEO}_{11} \mathrm{MA}\right)_{128^{-}} \\
b-\end{array}$ & 2 & 657 & 128 & $0.36 / 2.81$ & 107,000 \\
\hline \multicolumn{6}{|l|}{$\mathrm{PAA}_{657}$} \\
\hline $\mathrm{P}\left(\mathrm{PE}_{45} \mathrm{MA}\right)_{24}-b-$ & 3 & 566 & 24 & $0.49 / 2.02$ & 87,000 \\
\hline \multicolumn{6}{|l|}{$\mathrm{PAA}_{566}$} \\
\hline PAA & 4 & 130 & - & - & 13,000 \\
\hline $\mathrm{P}\left(\mathrm{PEO}_{11} \mathrm{MA}\right)_{76}$ & 5 & - & 76 & - & 36,000 \\
\hline
\end{tabular}

${ }^{\mathrm{a}}$ Ref. [15].

\section{Experimental section}

\subsection{Synthesis}

RAFT copolymerization of AA and poly(ethylene oxide)-methyl-ether-methacrylate (PEOMA) was carried out in butanol at $90{ }^{\circ} \mathrm{C}$ with bis(phenylmethyl) trithiocar-bonate as a RAFT chain transfer agent (CTA) and 4,4'azobis(4-cyanopentanoic acid) (V501) as an initiator. In a typical experiment, a mixture of V501 (0.1 equiv., 4.2 $\times 10^{-4} \mathrm{~mol}^{-1}$ ) and CTA ( 1 equiv., $4.2 \times 10^{-3} \mathrm{~mol}^{-1}$ ) was degassed by three nitrogen-vacuum cycles. Degassed acrylic acid (685 equiv., $2.88 \mathrm{~mol}^{-1}$ ), $n$-butanol and poly(ethylene oxide ${ }_{11}$ )-methyl-ether-methacrylate, $\mathrm{PEO}_{11} \mathrm{MA}$ (the number in subscript is the average degree of polymerization of ethylene oxide; 102 equiv., 0.43 mol $\mathrm{l}^{-1}$ ), were added at room temperature.

This synthesis was also repeated by substituting a poly(ethylene $\quad$ oxide $_{45}$ )-methyl-ether-methacrylate, $\mathrm{PEO}_{45} \mathrm{MA}$ (23 equiv., $0.1 \mathrm{~mol} \mathrm{l}^{-1}$ ) for $\mathrm{PEO}_{11} \mathrm{MA}$, under the same experimental conditions.

\subsection{Self-association in water}

Two distinct recipes were used to dissolve the copolymers in water. The first method consisted of the direct dissolution of the solid copolymer in $\mathrm{pH}$-adjusted water $(\mathrm{C}=16 \mathrm{~g} / \mathrm{I})$. In an alternative method, the copolymer was first dissolved in ethanol $\left(1.25 \times 10^{-2} \mathrm{~g}\right.$ of copolymer in $2.83 \mathrm{ml}$ of ethanol). The solution was then filtered through a membrane with a nominal pore size of $0.2 \mu \mathrm{m}$, added with $0.25 \mathrm{ml}$ of deionized water with a microsyringue, and dialyzed against water after stirring for $24 \mathrm{~h}$ (Spectra-Por membrane with cut-off of $3500 \mathrm{Da}$ ). The final concentration of the copolymer in aqueous solution was determined, and the $\mathrm{pH}$ was adjusted, if necessary, by using a $50 \mathrm{mM}$ phosphate buffer solution instead of deionized water. The ionic strength was determined by the addition of appropriate amounts of a $\mathrm{NaCl}$ solution when required.

\subsection{Dynamic light scattering (DLS)}

DLS measurements were performed with a Brookhaven Instruments Corp. BI-200 apparatus, equipped with a BI-2030 digital correlator and an Ion Laser Technology argon laser at a wavelength of $488 \mathrm{~nm}$. A refractive index matching bath of filtered decaline surrounded the scattering cell, and the temperature was controlled at $25^{\circ} \mathrm{C}$

The scattering angle used for the measurements was $90^{\circ}$, and the second-order correlation function, $\mathrm{G}_{2}(\mathrm{t})$, was measured [10].

In case of a single-exponential decay, $\mathrm{G}_{2}(\mathrm{t})$ can be expressed as

$$
G_{2}(t)=B[1+\beta \exp (-2 \Gamma t)]=B\left[1+\beta\left|G_{1}(t)\right|^{2}\right]
$$

where $B$ is the baseline, $\beta$ is an optical constant that depends on the instrument, $\Gamma$ is the decay rate for the process, $t$ is time, and $G_{l}\{t$ ) is the first-order correlation function, which was analyzed by the methods of the cumulants: 


$$
G_{1}(t)=\exp \left[-\Gamma_{1} t+\left(\frac{\Gamma_{2}}{2 !}\right) t^{2}-\left(\frac{\Gamma_{3}}{3 !}\right) t^{3}+\ldots\right]
$$

where $\Gamma_{\mathrm{i}}$ is the $i$ th cumulant. The polydispersity index (PDI) of the aggregates was estimated from the $\Gamma_{2} / \Gamma^{2}{ }_{1}$ ratio. The Z-average diffusion coefficient over the whole set of aggregates was calculated from the first cumulant:

$\Gamma_{l}=D q^{2}$

where $\mathrm{D}$ is the translation diffusion coefficient and $q$ is the absolute value of the scattering vector:

$$
q=\frac{4 \pi n \sin \left(\frac{\theta}{2}\right)}{\lambda}
$$

$n$ is the refractive index of the solvent, $\theta$ is the diffusion angle, and $\lambda$ is the wavelength of the incident light.

The diffusion coefficient extrapolated to zero concentration $\left(\mathrm{D}_{0}\right)$ for spherical particles is related to the hydrodynamic radius, $R_{h}$, by the Stokes-Einstein equation:

$$
D_{0}=\frac{k_{\mathrm{B}} T}{6 \pi \eta R_{\mathrm{h}}}
$$

where $k_{B}$ is the Boltzmann constant, $T$ is the absolute temperature, and $\eta$ is the solvent viscosity.

The mean hydrodynamic diameter $\left(\mathrm{D}_{\mathrm{h}}=2 R_{h}\right)$ and PDI of the aggregates were accordingly calculated. The DLS data were also analyzed by the CONTIN routine, a method based on a constraint inverse Laplace transformation of the data which gives access to a size distribution histogram for the aggregates.

Scheme 1. Structure of the investigated copolymers in which the length of the PEOMA grafts has been changed.

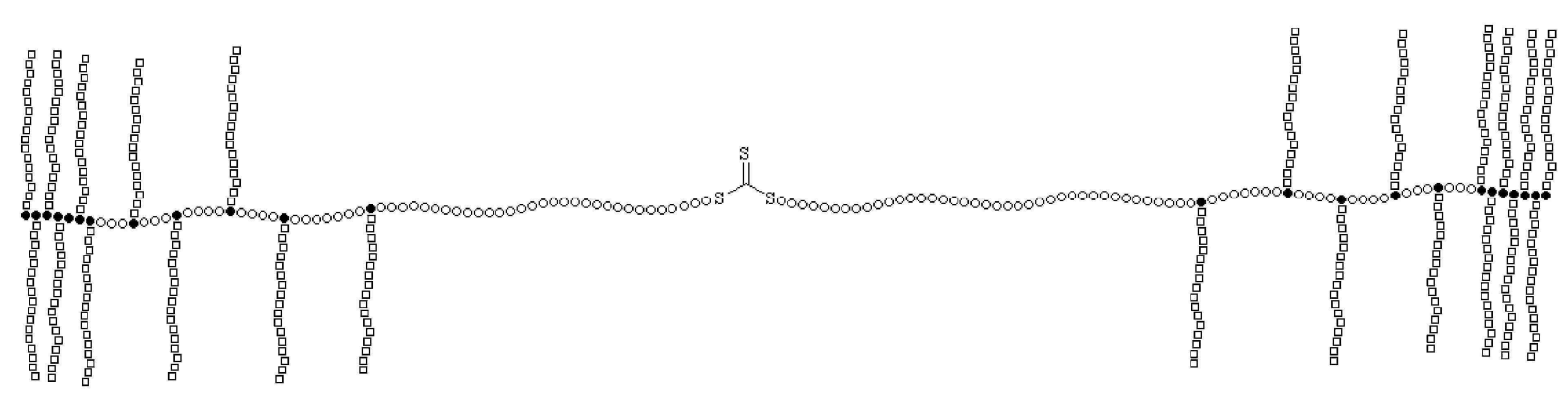

\subsection{Transmission electron microscopy (TEM)}

TEM images were recorded with a Philips CM 100 microscope equipped with a Gatan 673 CCD camera, and transferred to a computer equipped with the Kontron KS100 system. Samples were prepared by dipping a Formvar-coated copper grid into a dilute solution of the aggregates ( $2 \mathrm{wt} \%$ copolymer). Samples were negatively contrasted by depositing a drop of $1 \mathrm{wt} \%$ phosphotungstic acid aqueous solution onto the surface of the sampleloaded grid. 3 min later, the solution was blotted with a filter paper. The sample was finally washed with water, dried in air and contrasted by $\mathrm{RuO}_{4}$ vapor released by a solution of $\mathrm{RuCl}_{3}$ and $\mathrm{NaClO}(5 \mathrm{wt} \%$ ). Aqueous uranyl acetate was also used as a selective staining agent for the PAA blocks [11]. A drop of $1 \mathrm{wt} \%$ solution of the staining compound was deposited onto the surface of the sample-loaded grid, followed by drying in air.

\section{Results and discussion}

Reversible addition fragmentation chain transfer (RAFT) is a well-known mechanism for the controlled radical polymerization of water-soluble monomers [12]. Therefore, statistical copolymers of acrylic acid (AA) and poly(ethylene oxide) macromonomers [( $\left.\mathrm{PEO}_{\mathrm{x}}\right) \mathrm{MA}$, where $x$ is the average degree of polymerization of PEO] 
have been synthesized by RAFT, with dibenzyl trithiocarbonate as a difunctional transfer agent. The reactivity ratios for these comonomers have been determined elsewhere, by both the linearization methods of Mayo-Lewis [13] and Fineman-Ross [14], respectively. The two methods agreed on the following data, i.e. 2.02/0.49 for the $\mathrm{PE}_{45} \mathrm{MA} / \mathrm{AA}$ pair and 2.81/0.36 for the $\mathrm{PEO}_{11} \mathrm{MA} / \mathrm{AA}$ one [15]. Because the product of the reactivity ratios is close to one, the copolymerization is non-azeotropic ideal and the comono-mer distribution must be of the blocky-type. As result of the difunctionality of the chain transfer agent, formation of pseudo-triblock copolymer chains is predictable, with a tapered structure consisting of an inner PAA block and two outer comb-like blocks of $\mathrm{P}\left(\mathrm{PEO}_{\mathrm{x}} \mathrm{MA}\right)$ as illustrated in Scheme 1 [16].

Three copolymers have been synthesized, and their molecular characteristic features are reported in Table 1, together with those ones for the parent homopolymers, PAA and P(PEOMA), synthesized under the same experimental conditions.

These copolymers are typically double hydrophilic copolymers [1], with possible hydrogen bonding of the constitutive blocks, thus between the carboxylic acid groups of PAA and the oxygen atoms of PEO. These secondary interactions are known for high sensitivity to $\mathrm{pH}$, temperature and ionic strength $[5,9]$. These experimental parameters have also a direct effect on the water solubility of PAA and PEO. Indeed, the properties of the aqueous solutions of PAA depend on $\mathrm{pH}$ and ionic strength [17], whereas solvation of PEO depends on temperature [18] and ionic strength [19]. Self-assemblies of the blocky copolymers listed in Table 1 are thus expected to be stimuli-responsive whenever dissolved in water.

All the synthesized homopolymers and copolymers (Table 1) have been tentatively dissolved into $\mathrm{pH}$-adjusted water. The polymacromonomer, $\mathrm{P}(\mathrm{PEOMA})$, is soluble in the whole $\mathrm{pH}$ range, in contrast to PAA which is only soluble at $\mathrm{pH}>5$. The three copolymers are insoluble at $\mathrm{pH}<5$, poorly soluble at $5<\mathrm{pH}<8$, and soluble at $\mathrm{pH}>8$. In all these solubility tests, the bulk copolymers have been directly added to the aqueous phase, without shaking, ageing or heating. The aqueous solutions have been analyzed by dynamic light scattering $(5 \mathrm{~g} / 1$. Large aggregates were detected for the aqueous solutions of $\mathrm{P}(\mathrm{PEOMA})$ in the whole $\mathrm{pH}$ range, for PAA at high $\mathrm{pH}$ and for the three copolymers at $\mathrm{pH}>8$. Because of limited solubility of the copolymers, no reliable DLS data could be collected in the $5<\mathrm{pH}<8$ range. As a rule, the light scattered by all these aqueous solutions is low, consistent with formation of loose aggregates. This observation is not surprizing, because polyelectrolytes are known to form loose aggregates in aqueous solution [20], which is also reported for PEO [21]. The PEO and PAA blocks could participate to intra- and/or intermolecular hydrogen bonding in the bulk, so being a brake to solubilization and leading to out-of-equilibrium structures. In order to overcome the problem of the low amount of copolymers directly dissolved in water at $5<\mathrm{pH}<8$, thus under conditions required for the occurrence of hydrogen-bonding between non ionized acid and ethylene oxide units, the copolymers have been first dissolved in ethanol, in which preformed hydrogen-bonding and related aggregation are minimized compared to water, followed by the dropwise addition of $\mathrm{pH}$-adjusted water until stable self-aggregation is observed and final dialysis against $\mathrm{pH}-$ controlled water. This preparation method is quite similar to the one previously reported by Eisenberg et al. [22].

Once again, whenever the $\mathrm{pH}$ is lower than 5, the copolymers are poorly soluble, which is not very surprizing because most of the AA units are in the acidic form and contribute extensively to hydrogen-bonding.

Eventhough intramolecular H-bonds are favoured in dilute solution, intermolecular bonding cannot be precluded and be responsible for the poor solubility at low $\mathrm{pH}$. Once again the effect of shaking, ageing and heating has not been investigated. That hydrogen-bonding is at the origin of aggregation has been confirmed by the quasi complete disappearance of aggregation upon addition of a large excess of urea $(50 \mathrm{~g} / 1)$ at $5<\mathrm{pH}<8$, in agreement with previous reports on similar systems [7].

Stable aggregates are observed in the $5<\mathrm{pH}<8$ range. A high scattering intensity is observed by DLS, which suggests formation of densely packed polymer phases with a refractive index substantially different from the surrounding aqueous phase. Loose aggregates responsible for very low scattering intensity are again observed at $\mathrm{pH}>8$.

The aqueous solutions of the three copolymers have been analyzed more carefully in the $5<\mathrm{pH}<8$ range by DLS. Self-assemblies with a $D_{h}$ in agreement with traditional micellar structures are observed for copolymer 2 (Table 2). Larger objects are formed by copolymers 1 and 3. The size of the aggregates of copolymer 1 at $\mathrm{pH}=5$ is too large for being consistent with spherical micelles made of a dense core and a corona of solvated chains.

Nevertheless, the angular dependence of the diffusion coefficient confirms that these aggregates have a spherical shape [23].

The CONTIN routine has been used to analyze the size distribution of the copolymer aggregates. Measurements have been repeated five times and found reproducible. The CONTIN size distribution histograms are shown in Fig. 1 for the three copolymers at $\mathrm{pH}=7$. Distributions are monomodal and narrow for copolymers 1 and 2 . 
Published in : Polymer (2004), vol. 45, iss. 25, pp. 8303-8310

Status : Postprint (Author's version)

Fig. 1. Histograms for the CONTCN size distribution of the copolymer self-assemblies ( $p H=7, C=4 \mathrm{~g}^{-1}$ ).

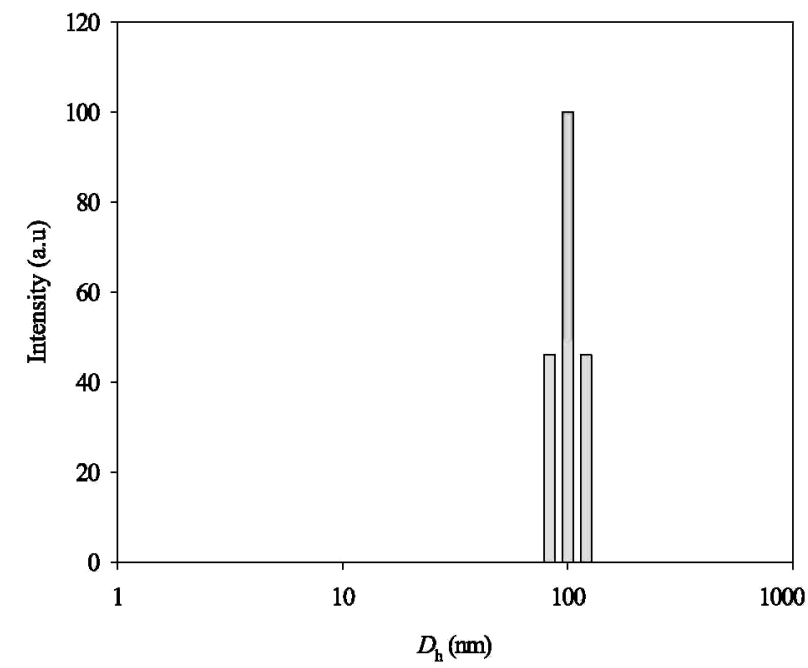

Copolymer 1

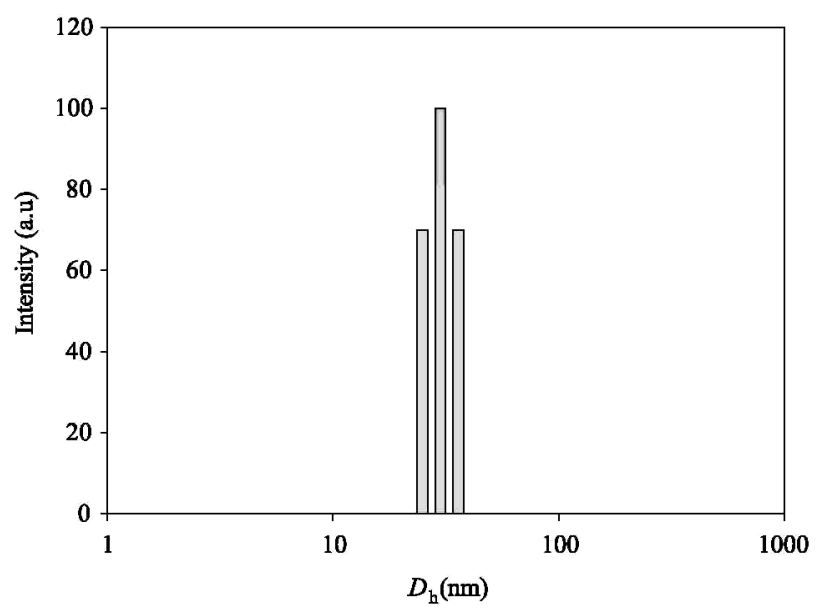

Copolymer 2

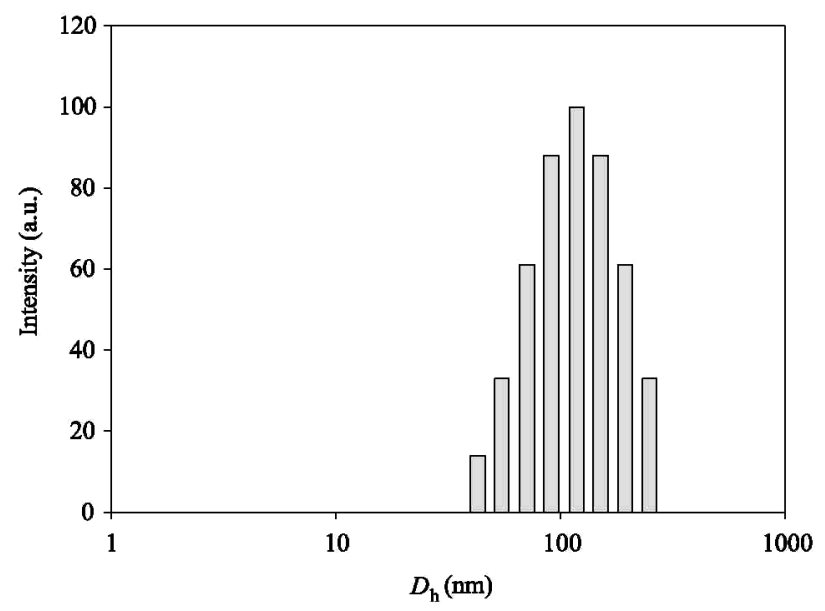

Copolymer 3 
Table 2 Average size of the copolymer self-assemblies formed in water at different $p H$-DLSdata $(C=16 \mathrm{~g} / \mathrm{l})$

\begin{tabular}{llll}
\hline Copolymer & \multicolumn{1}{|l}{$\mathbf{D}_{\mathbf{h}}(\mathbf{n m})$} & & \\
\cline { 2 - 4 } & $\mathbf{p H}=\mathbf{5}$ & $\mathbf{p H}=\mathbf{7}$ & $\mathbf{p H}=\mathbf{l 0}$ \\
\cline { 2 - 4 } 1 & 321 & 96 & 264 \\
2 & 31 & 31 & 278 \\
3 & 95 & 131 & 454
\end{tabular}

More detailed information on the morphology of the copolymer aggregates has been searched for by transmission electron microscopy (TEM). Fig. 2 shows a TEM micrograph for the aggregates formed by copolymer 2 at $\mathrm{pH}=7$. The aggregates are observed as white dots on a dark background as result of a negative contrast by uranyl acetate. They are spherically shaped, with an average diameter $(30 \mathrm{~nm})$ in agreement with DLS data. Spherical aggregates are also observed for copolymer 3 (Fig. 3), that have sizes quite comparable to copolymer 2 although with a higher polydispersity. The larger objects have a diameter of $50 \mathrm{~nm}$, thus much lower than the size measured by DLS (Table 2), which cannot be explained yet.

Fig. 4 shows the spherical aggregates formed by sample 1, as observed by TEM. Their characteristic size is in good agreement with DLS measurements. Fig. 4 shows that the aggregates consist of ring in which the contrasting agent has accumulated, around a core with an electronic density comparable to the background. The aggregates thus appear as hollow capsules. Very similar TEM pictures have been recently reported for aggregates formed by double hydro-philic copolymers of PAA and hydroxyethylcellulose at neutral $\mathrm{pH}$. They were accounted for by the formation of hollow spheres (see Fig. 2(d) in Ref. [24]). In these copolymers, the PAA chains are grafted onto a hydroxyethylcellulose backbone, and they are the major constituent as is the case for sample 1 .

Fig. 2. TEM picture of self-assemblies of copolymer 2 at $\mathrm{pH}=7\left(\mathrm{C}=16 \mathrm{~g} \mathrm{I}^{-1}\right)$ Negative contrast by uranyl acetate.

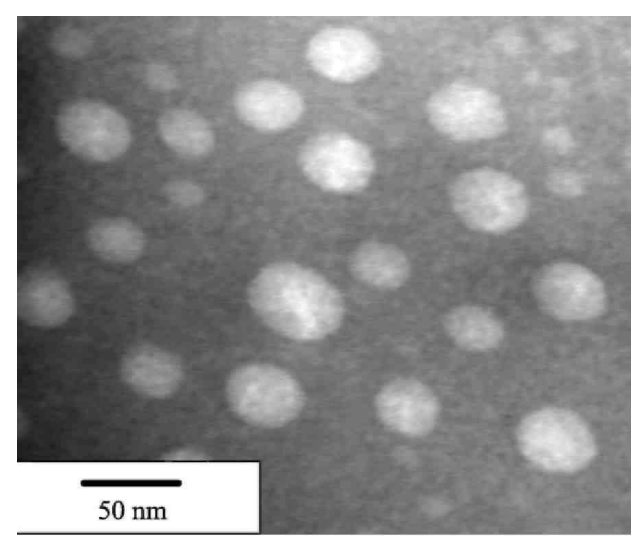

Fig. 3. TEM pictureofself-assembliesofcopolymer3atpH $=7\left(C=4 \mathrm{~g} I^{-1}\right)$. Negative contrast by uranyl acetate.

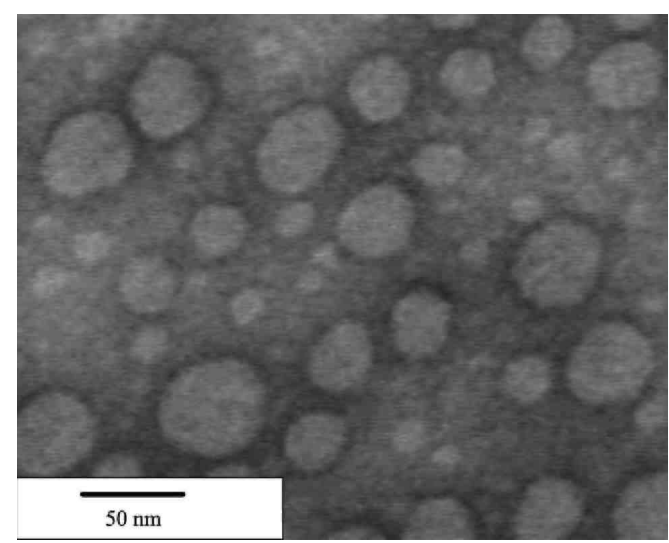


Fig. 4. TEM picture of self-assemblies of copolymer 1 at $p H=7\left(C=16.5 \mathrm{~g}^{-1}\right)$. Staining by phosphotungstic acid.

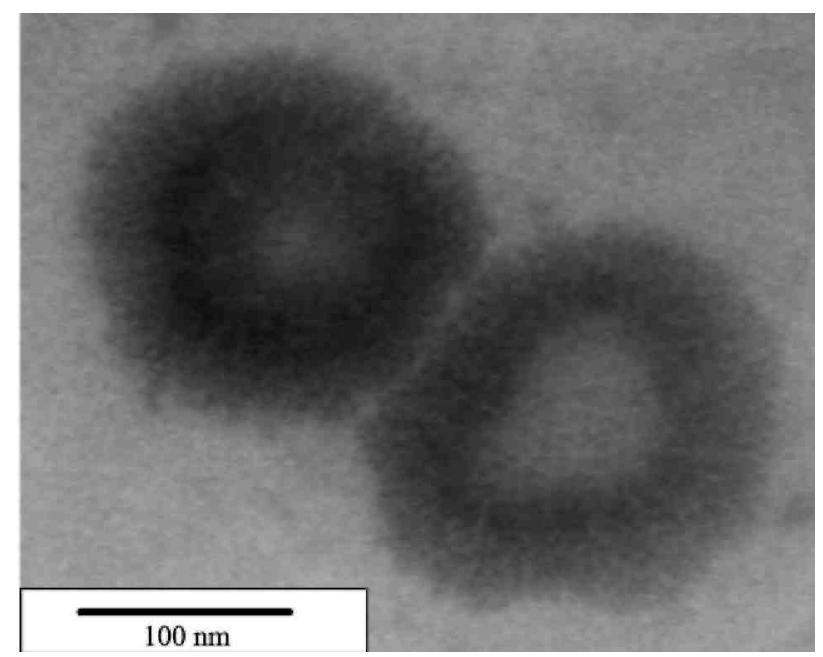

Composition of the copolymer has been changed and the length of the PEO branches, as well. Samples 2 and 3 have a similar EO/AA molar ratio $(\sim 2)$ but the PEO grafts are of a different length (Table 1). The EO/AA ratio is much lower (0.37) in sample 1, which means a higher content of AA units, and the PEO grafts are the same as in sample 2. Dense spherical aggregates are formed by samples 2 and 3, whereas hollow particles are observed in case of sample 1. From this comparison of three samples and within the limits of their molecular characteristics, it appears that the structure of the copolymer self-assemblies is dominated by the EO/AA ratio and not by the length of the PEO branches. This observation is consistent with the mutual interactions of the AA and EO units by hydrogen-bonding. Nevertheless, the colloidal particles are only stable in a finite range of $\mathrm{pH}$, i.e., whenever a significant fraction of the AA units are ionized and can contribute to the self-aggregate stabilization by an electrosteric mechanism [25]. In order to check this hypothesis, additional experiments have been carried out, in which ionic strength and temperature have been changed.

Salt $(\mathrm{NaCl})$ has been added to the aqueous solutions of the copolymers, in the $5<\mathrm{pH}<8$ range. DLS data have been monitored as a function of the added salt concentration. As a rule, the same behavior is observed for the three copolymers, as illustrated in Fig. 5 for copolymer 2. In the salt concentration range lower than 0.5 mol, $D_{h}$ of the self-assemblies remain constant. Above this critical salt concentration, a sharp increase in the characteristic dimension of the aggregates is noted, together with a sharp decrease in the scattered light intensity. A deaggregation phenomenon thus occurs which results in much looser structures, whenever a sufficient amount of salt is added. TEM observations confirm that well-structured aggregates are only observed below a certain salt concentration. At high concentration, macroscopic precipitation occurs with time. Screening of the ionized AA units by $\mathrm{NaCl}$ must have a very deleterious effect on the electrosteric stabilization of the aggregates. Moreover, possible complexation of $\mathrm{Na}$ cations by EO units could compete the EO/AA pairing by hydrogen-bonding [26]. Finally, the water solubility of PEO is adversely affected by a salt addition, possibly leading to the PEO precipitation [19].

The effect of temperature has also been analyzed in the $5<\mathrm{pH}<8$ range. A previous work on poly(methacrylic acid)-block-poly(ethylene oxide) copolymers showed the formation of micelles at low $\mathrm{pH}$ and room temperature, whose the core consisted of EO/MAA hydrogen-bonded complexes [7]. Whenever the temperature was raised up to $45^{\circ} \mathrm{C}$, the hydrogen-bonded complexes were disrupted, and a new type of micelles was formed with an insoluble PMAA core and a corona of uncomplexed PEO blocks. According to the data in Figs. 6 and 7, a similar behavior is observed for copolymer 3 . An increase in both $D_{h}$ and scattered intensity is indeed observed at approximately $50{ }^{\circ} \mathrm{C}$. This transition is thermoreversible and not accompanied by a simultaneous transition from transparent to opalescent solution. Thus, precipitation even minor does not occur. The plateau in the scattered intensity above $\sim 60{ }^{\circ} \mathrm{C}$ is consistent with the stability of the aggregates in this domain. The effect of temperature would thus be a weakening of the hydrogen-bonded EO/AA complexes making a structural reorganization possible. Furthermore, the solubility of PEO is decreased upon increasing temperature [18]. Although the temperature did not exceed LCST of PEO in this work, a time-dependent flocculation of the aggregates has been observed at high temperature, consistent with the slow desolvation of the PEO chains.

In sharp contrast, temperature has no influence on the characteristic sizes of the aggregates formed by 
copolymers 1 and 2, in the $5<\mathrm{pH}<8$ range. This is typically illustrated by copolymer 2 at $\mathrm{pH}=7$ (Fig. 8). These two copolymers contain short PEO grafts $(\mathrm{DP}=11)$, whose the (partial) complexation with PAA segments contributes to the formation of the cores of the aggregates. The self-assembled structure remains essentially unmodified upon EO/AA complexation at increasing temperatures. When longer PEO grafts are involved, the structure is reorganized, which illustrates the complexity of the self-association of the double hydrophilic copolymers under consideration.

For sake of comparison, solubility of both the $\mathrm{P}\left(\mathrm{PEO}_{11} \mathrm{MA}\right)$ homopolymer (sample 5, Table 1) and the PAA homopolymer (sample 4, Table 1) has been investigated in relation to $\mathrm{pH}$. $\mathrm{P}\left(\mathrm{PEO}_{11} \mathrm{MA}\right)$ is soluble in the whole $\mathrm{pH}$ range with formation of loose aggregates with a $D_{h}$ of $\sim 265 \mathrm{~nm}$, as measured by DLS. PAA is completely soluble in water at $\mathrm{pH}>5$. When a $50 / 50 \mathrm{wt} / \mathrm{wt}$ blend of the two homopolymers is concerned, poor solubility is noted at $\mathrm{pH}<5$, whereas loose aggregates with a very large size (DLS) are observed at higher $\mathrm{pH}$, which is confirmed by TEM. The difference in the association of the triblock copolymers and PAA/PEO mixture can be accounted for by the intra- versus intermolecular hydrogen-bonding. Only intermolecular bonding is possible between PAA and PEO in the mixtures, which results in ill-defined structures. In case of dilute solutions of triblock copolymers, intramolecular interactions should dominate, quite similarly for all the copolymer chains which can accordingly self-assemble in well-defined structures. This situation is quite reminiscent of the interpolyelectrolyte complexation of oppositely charged blocks that form well-defined aggregates [27]. The copolymer architecture is thus a prerequesite for the formation of structured aggregates.

Fig. 5. Influence of $\mathrm{NaCl}$ concentration ( $\left.m o l 1^{-1}\right)$ on $D_{h}$ of self-assemblies of copolymer 3 at $p H=7\left(C=16 g 1^{-1}\right)$.

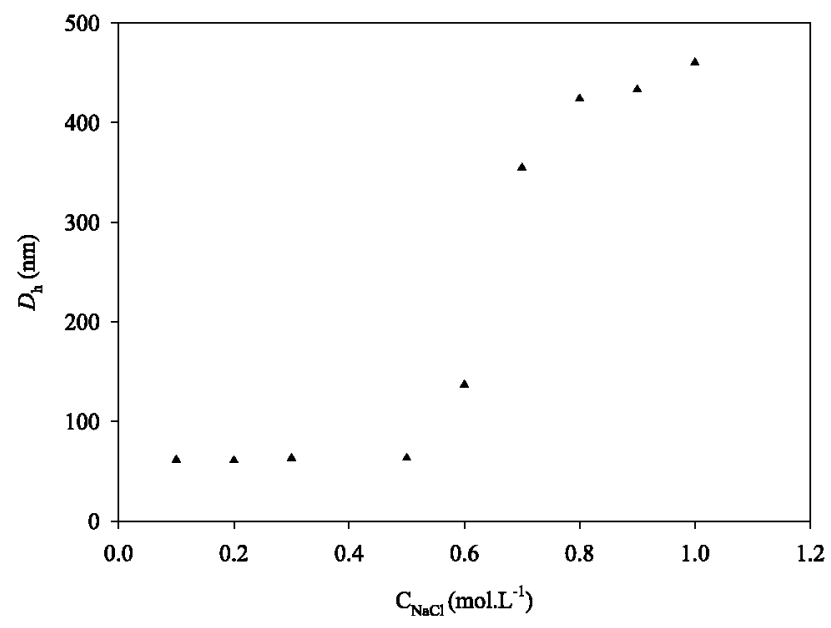

Fig. 6. Influence of temperature on the DLS data for the self-assemblies of copolymer 3 at $p H=7\left(C=4 g 1^{-1}\right)$.

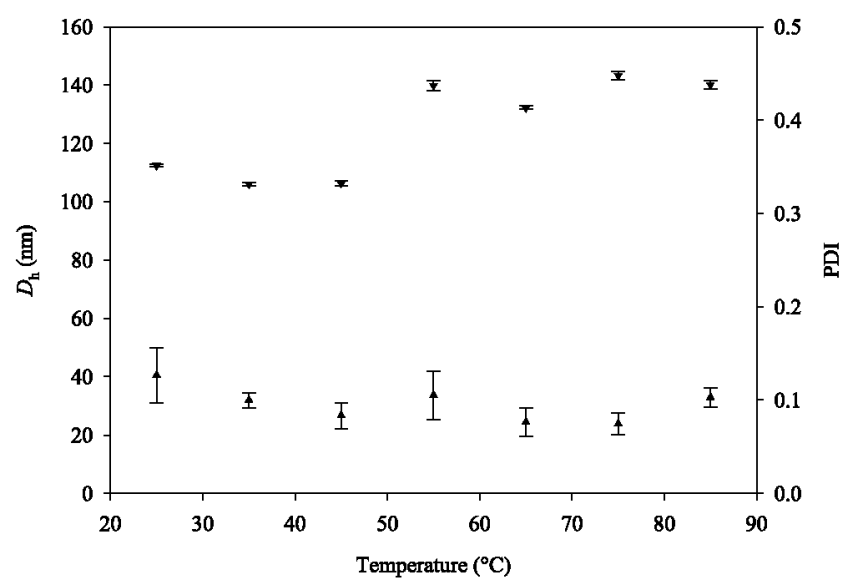


Fig. 7. Influence of temperature on the scattered light intensity (in counts per second, cps) for the selfassemblies of copolymer 3 at $\mathrm{pH}=7\left(\mathrm{C}=16 \mathrm{~g}^{-1}\right)$.

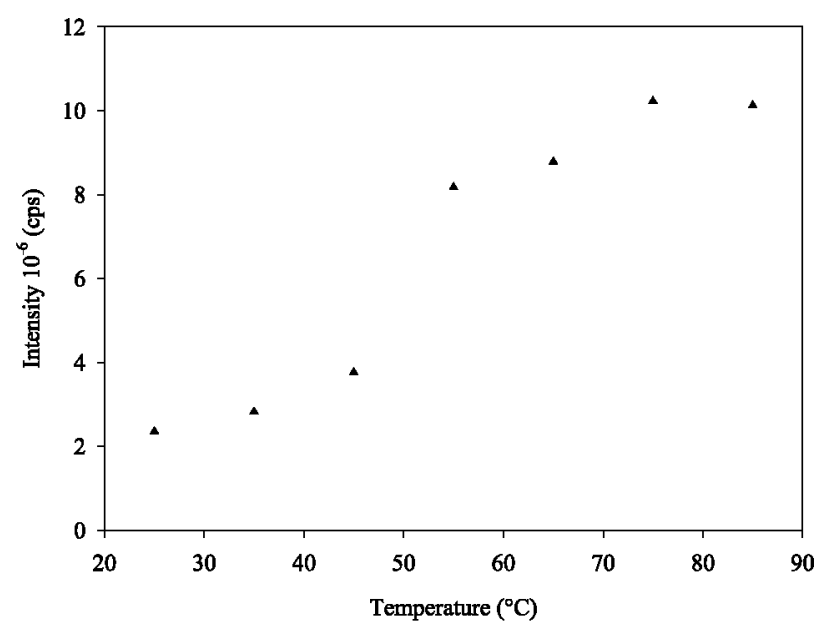

Fig. 8. Influence of temperature on the DLS data for the self-assemblies of copolymer 2 at $p H=7\left(C=16 \mathrm{~g} \mathrm{I}^{-1}\right)$.

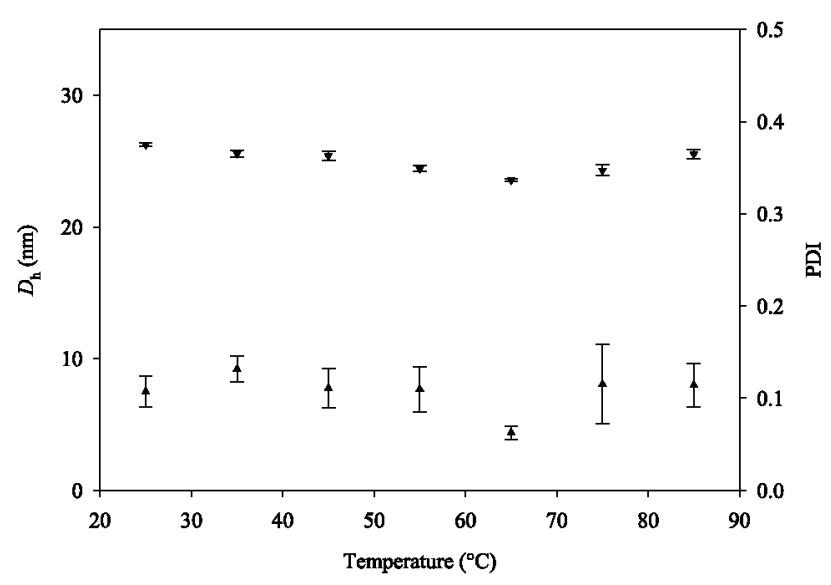

\section{Conclusions}

Double-hydrophilic tapered P(PEOMA)- $b$-PAA- $b$-P(PEOMA) copolymers of different composition and length of PEOMA grafts have been synthesized and their behavior in water has been investigated. Solutions have been prepared by dissolution in ethanol, followed by dialysis against water. At $\mathrm{pH}<5$, the copolymers are poorly soluble in water. In the $5<\mathrm{pH}<8$ range, polydispersed spherical dense aggregates are observed by DLS and TEM for the copolymers that contain the higher EO/AA molar ratio $(\sim 2)$. When this ratio is reversed $(\sim 0.37)$, hollow spheres are formed rather than the spherical dense nanoobjects, which shows the key role of this structural parameter. It must be noted that the constitutive PAA and P(PEOMA) chains must be chemically bonded one to each other for structured aggregates to be formed. Indeed, a mixture of the two forms loose aggregates under the same conditions. At $\mathrm{pH}>8$, loose aggregates of an ill-defined structure are systematically observed. The aggregates formed in the $5<\mathrm{pH}<8$ range can however be disassembled by addition of a sufficient amount of salt. Increasing temperature from 25 to $85^{\circ} \mathrm{C}$ has no significant effect whenever the copolymer contain short PEO grafts $(\mathrm{DP}=11)$. In case of longer PEO grafts $(\mathrm{DP}=47)$, a reversible transition to another type of aggregates is observed at $\sim 50{ }^{\circ} \mathrm{C}$. These observations strongly support that the driving force for self-assembly is the formation of hydrogen bonds between the AA and EO units, the aggregates being stabilized by the electrosteric repulsion of ionized PAA segments. 


\section{Acknowledgements}

EK and RJ are very much indebted to the 'Belgian Science Policy' for financial support in the frame of the Tnteruniversity Attraction Poles Programme (PAI V/03 - Supramolecular Chemistry and Supramolecular Catalysis)'. JFG is grateful to the Communauté française de Belgique (ARC 03/08-300, NANOMOL).

\section{References}

[1] Cölfen H. Macromol Rapid Commun 2001;22:219.

[2] Martin TJ, Prochakza K, Munk P, Webber SE. Macromolecules 1996;29:6071.

[3] Bekturov EA, Bimendina LA. Adv Polym Sci 1981;41:99.

[4] Tsuchida E, Abe K. Adv Polym Sci 1982;45:1.

[5] Mathur AM, Drescher B, Scranton AB, Klier J. Nature 1998;392:367.

[6] Robinson DN, Peppas NA. Macromolecules 2002;35:3668.

[7] Gohy JF, Varshney SK, Jérôme R. Macromolecules 2001;34:3361.

[8] Eliassaf J, Silberberg A. Polymer 1962;3:555.

[9] Holappa S, Karesoja M, Shan J, Tenhu H. Macromolecules 2002;35:4733.

[10] Gohy JF, Varshney SK, Antoun S, Jérôme R. Macromolecules 2000;33:9298.

[11] Schuch H, Klinger J, Rossmanith P, Frechen T, Gerst M, Feldthusen J, Müller AHE. Macromolecules 2000;33:1734.

[12] see for example: (a) Arotçaréna M, Heise B, Ishaya S, Laschewsky A. J Am Chem Soc 2002; 124:3787.

(b) Taton D, Wilczewska AZ, Destarac M. Macromol Rapid Commun 2001 ;22:1497.

(c) Gaillard N, Guyot A, Claverie J. J Polym Sci Chem Ed 2003 ;A41 : 684.

[13] Mayo FR, Lewis FM. J Am Chem Soc 1944;66:1594.

[14] Fineman M, Ross SD. J Polym Sci 1950;5:259.

[15] Khousakoun E, Jérôme R. Submitted for publication.

[16] Lai JT, Filla D, Shea R. Macromolecules 2002;35:6754.

[17] Dautzenberg H, Jaeger W. Polyelectrolytes formation, characterization and application. New York: Hanser; 1994.

[18] Mortensen K. J Phys Condens Matter 1996;8:103.

[19] Jain NJ, Aswal VK, Goyal PS, Bahadur P. Colloids Surf A: Physicochem Eng Aspects 2000;173:85.

[20] Förster S, Schmidt M, Antonietti M. Polymer 1990;31:781.

[21] Duval M, Sarazin D. Polymer 1999;41:2711.

[22] Zhang L, Eisenberg A. Science 1995;268:1728.

[23] Pecora RJ. J Chem Phys 1968;48:4126.

[24] Dou H, Jiang M, Peng H, Chen D, Hong Y. Angew Chem Int Ed 2003 ; 42:1516.

[25] Napper DH. Polymeric stabilization of colloidal dispersions. London: Academic Press; 1983.

[26] Sartori R, Sepulveda L, Quina F, Lissi E, Abuin E. Macromolecules 1990;23:3878.

[27] Gohy JF, Creutz S, Garcia M, Mahltig B, Stamm M, Jérôme R. Macromolecules 2000;33:6378. 